GEOMETRY IN NONLINEAR CONTROL

AND DIFFERENTIAL INCLUSIONS

BANACH CENTER PUBLICATIONS, VOLUME 32

INSTITUTE OF MATHEMATICS

POLISH ACADEMY OF SCIENCES

WARSZAWA 1995

\title{
SINGULARITIES OF THE MAXIMUM FUNCTION OVER A PREIMAGE
}

\author{
ALEKSEY DAVYDOV \\ Department of Mathematics, Vladimir State Technical University \\ Gorkii street 87, 600026 Vladimir, Russia
}

1. Introduction. In this paper generic singularities of maximum (minimum) functions over a preimage are classified in the case of one-dimensional or twodimensional image-space, and preimage-space of dimension not smaller than that of the image-space. It turns out that in the case of general position there are four and thirteen singularities of such functions respectively (up to $\Gamma$-equivalence defined below). In the first case their list does not depend on the dimension of the preimage-space and in the second one it stabilizes starting from dimension three. To be more precise, on a smooth (i.e. of class $C^{\infty}$ ) manifold $N$ without boundary we consider a pair of smooth mappings, namely, a function $g: N \rightarrow R_{t}^{1}$ and a proper mapping $\tau: N \rightarrow R_{q}^{m}$. On the image of the mapping $\tau$ the pair defines the maximum function over the preimage $G(q)=\max \left\{g(p) \mid p \in \tau^{-1}(q)\right\}, q \in \tau(N)$. We endow the space of such pairs with the Whitney fine $C^{\infty}$-topology. A pair in general position or a generic pair is a pair lying in some open dense set. The generic singularities of maximum functions over preimages are the singularities of those functions for generic pairs. These singularities cannot be removed by small perturbations of the pair.

It is easy to see that $G(q)$ is a solution of the elementary smooth problem $q(p) \rightarrow$ max under the constraint $\tau(p)=q$ [5]. Thus the classification of the singularities of maximum functions over preimages also provides a list of generic singularities of the solution of such a problem as a function of $q$ (in particular, a list of generic singularities of the boundary of the set of values of $q$ where the function $G$ is defined).

By changing the sign of the function $g(G)$, all the results obtained for max-

1991 Mathematics Subject Classification: 49B10, 93C10.

Research was partially suported by AMS grant and Intern. Science Found. grant MSD000.

The paper is in final form and no version of it will be published elsewhere. 
imum functions can be extended to the case of minimum functions. The generic singularities of the latter for $\operatorname{dim} N>m=2$ can be realized as the singularities of the time optimal function of a two-dimensional control system (such a realization of one of the singularities is given in Section 2). The relationship between the singularities of wave fronts and those of the minimum function of the family of functions is well known [2], [3], [14]. It seems to have appeared for the first time in [12].

The classification of the generic singularities of maximum and minimum functions over preimages gives an opportunity to classify the generic singularities of the field of limit directions of a smooth differential inequality $F(z, \dot{z}) \leq 0$ on a surface with locally bounded derivatives. The latter classification proves to be important for the investigation of integral funnels for such inequalities. That classification will be treated in another paper. Some results concerning this classification have already been announced in [7].

If $\tau$ is a fiber map the list of generic singularities of maximum (minimum) functions over preimages (up to $R^{+}$-equivalence, i.e. up to a diffeomorphism of the image-space and addition of a smooth function defined on this space) is finite for $m \leq 6$. It was found by L. N. Bryzgalova [6]. In that case she also found all simple and stable germs of such a function for any $m$. Later V. I. Matov proved that the generic maximum (minimum) function over the preimage is topologically a Morse function [10].

I am grateful to V. I. Arnol'd, V. V. Goryunov and V. M. Zakalyukin for useful discussions concerning this work.

2. Classification of singularities. We start with the formulations of the main results of this paper.

2.1. Singularities of a maximum function over the preimage. A pair $(g, \tau)$ is called $I \Gamma$-stable if for any pair $(\tilde{g}, \tilde{\tau})$ sufficiently close to it the images of the manifold $N$ under the mappings $(g, \tau)$ and $(\tilde{q}, \tilde{\tau})$ can be transformed into one another by a $C^{\infty}$-diffeomorphism of the space $R_{q}^{m} \times R_{t}^{1}$ that is fibered over $R_{q}^{m}$ and close to the identity. Denote $n=\operatorname{dim} N$.

TheOREM 1. For $m=1$ or $m=2$ and $n \geq m$ the generic pair is $I \Gamma$-stable.

The germs of two functions are $\Gamma$-equivalent if there exists a $C^{\infty}$-diffeomorphism of the space of graphs of these functions which is fibered over the spaces in which they are defined and transforms one of these germs into the other.

THEOREM 2. If $n \geq m=1$ and if the pair is in general position, the germ at each point of the image of the maximum function over the preimage is $\Gamma$ equivalent to the germ at zero of one of the following four functions:
(1) 0 ;
(2) $|x|$;
(3) $\sqrt{x}$;
(4) $\max \{\sqrt{x} ;-1\}$ 
Here and further, unless otherwise specified, $x$ and $x, y$ are coordinates in $R_{q}^{1}$ and $R_{q}^{2}$ respectively.

THEOREM 3. If $n \geq m=2$ and if the pair is in general position, the germ at each point of the image of the maximum function over the preimage is $\Gamma$ equivalent to the germ at zero of one of the following thirteen functions:

(1) 0 ;

(8) $\max \{\sqrt{x} ; \sqrt{y}-1 ;-2\}$;

(2) $|x|$;

(9) $\max \{\sqrt{x} ; y\}$;

(3) $\sqrt{x}$;

(10) $\max \left\{\omega \in R \mid \omega^{3}+\omega x+y=0\right\}$;

(4) $\max \{\sqrt{x} ;-1\}$;

(11) $\max \left\{-\omega^{4}+\omega^{2} x+\omega y \mid \omega \in R\right\}$;

(5) ||$x|+y|+|x|$;

(12) $\sqrt{x}|y|$;

(6) $\max \{\sqrt{x} ;|y|-1\}$;

(13) $\max \{\sqrt{x}|y| ;-1\}$.

(7) $\max \{\sqrt{x} ; \sqrt{y}-1\}$;

The first ten singularities can be observed for any $n \geq 2$, the eleventh singularity may only occur when $n \geq 3$, and the last two singularities can only be met when $n=2$.

Endow the space $C^{\infty}(N, R)$ and the space $C_{p}^{\infty}\left(N, R^{m}\right)$ of proper mappings with the Whitney fine $C^{\infty}$-topology. The mapping in general position is a mapping from a certain open dense subset of the appropriate space of mappings in that topology.

THEOREM 4. For $m=1$ or $m=2$ and $n \geq m$, for a fixed mapping $g$ with Morse critical points (for an LR-stable mapping $\tau$ respectively) and for the mapping $\tau$ ( $g$ respectively) in general position the statements of Theorem 2 are valid if $m=1$, the statements of Theorem 3 are valid if $m=2$ and the statements of Theorem 1 are true.

Theorems 1-4 will be proved in Section 3.

R e m arks. 1. The $L R$-stability is the stability relative to the ordinary leftright equivalence [4], [9].

2. For $m=n=2$ part of the singularities from the list of Theorem 3 can be obtained from the normal forms of germs of the diagrams $R \leftarrow N \rightarrow R^{2}$ [1], [4], [8]. The other singularities from this list are transversal "overlappings" of the singularities obtained in this way.

2.2. Realization of singularities. It was noted above that when $n>m=2$ the generic singularities of minimum functions over preimages can be realized as generic singularities of the time optimal function in a two-dimensional control system. We give here a realization of singularities of type (10) from the list of Theorem 3 whose appearance as a singularity of a time optimal function is less obvious than that of the other ten singularities. 
Consider a control system in the plane defined by the equations $\dot{x}=\cos u$, $\dot{y}=x+\sin u$, where $u \in[0,2 \pi)$. At every point of the domain $x>1$ the directions of the admissible velocities form an angle smaller than $180^{\circ}$. The limit velocities lying on the sides of this angle are defined by values of the control parameter which are solutions of the equation $\sin u=-1 / x$. Hence $\cos u= \pm \sqrt{1-1 / x^{2}}$ and the family of integral curves of the field of limit velocities is defined by the equation $y^{\prime}= \pm \sqrt{1-x^{2}}$. By integration we find that $y= \pm\left(x \sqrt{x^{2}-1}-\ln (x+\right.$ $\left.\left.\sqrt{x^{2}-1}\right)\right) / 2+c$. For the initial set we take a circle $x=a+r \cos \phi, y=\sin \phi$, $0 \leq \phi<2 \pi, a>1$, touching at a certain point $A$ the curve $l: y=-\left(x \sqrt{x^{2}-1}-\right.$ $\left.\ln \left(x+\sqrt{x^{2}-1}\right)\right) / 2$. This curve passes through the boundary point $(1,0)$ of the domain of definition of the field of limit velocities. We shall show that at this point the time optimal function has a singularity of type (10) from the list of Theorem 3 up to $R^{+}$-equivalence. To do this, we use the Pontryagin Maximum Principle [13]. We introduce a vector $\left(\Psi_{1}, \Psi_{2}\right)$ and a function $H=\Psi_{1} \cos u+\Psi_{2}(x+\sin u)$. This vector satisfies the system of equations $\dot{\Psi}_{1}=-\Psi_{2}, \dot{\Psi}_{2}=0$ and is perpendicular to the initial set at time $t=0$. Consequently, $\left(\Psi_{1}(0), \Psi_{2}(0)\right)= \pm(\cos \phi, \sin \phi)$ if the motion begins from the point corresponding to the angle $\phi$. For the point $(1,0)$ and for any point sufficiently close to it the respective time optimal motion begins along the smaller arc $A B$, where $B=(a-r, 0)$, of the initial set with admissible velocity directed outside the initial set, as we can easily see. From this and from the condition $\max \{H \mid 0 \leq u<2 \pi\} \geq 0$ we find that this motion starts with the vector $\left(\Psi_{1}(0), \Psi_{2}(0)\right)=(\cos \phi, \sin \phi)$. Consequently, $\Psi_{1}(t)=-t \sin \phi+\cos \phi$ and $\Psi_{2}(t)=\sin \phi$. The time optimal control satisfies the equation $-\sin u(-t \sin \phi+$ $\cos \phi)+\cos u \sin \phi=0$ or $\operatorname{ctg} u=-t+\operatorname{ctg} \phi$ since it yields a maximum with respect to $u$ for the function $H$. It follows that the time optimal extremals for points sufficiently close to $(1,0)$ are defined by

$$
\begin{aligned}
& x(t)=a+r \cos \phi+\int_{0}^{t}(\tau \theta-\operatorname{ctg} \phi)\left(1+(\theta-\operatorname{ctg} \phi)^{2}\right)^{-1 / 2} d \theta, \\
& y(t)=r \sin \phi+\int_{0}^{t}\left(x(\theta)-\left(1+(\theta-\operatorname{ctg} \phi)^{2}\right)^{-1 / 2} d \theta .\right.
\end{aligned}
$$

The extremal goes to the point $(1,0)$ from the point $A$ and arrives with the value $u=-\pi / 2$ of the control parameter. Consequently, the time of arrival is $t_{0}=\operatorname{ctg} \phi_{0}$, where $\phi_{0}$ is the angle corresponding to the point $A$. It is easy to find that: $x_{\phi}\left(t_{0}, \phi_{0}\right)>0, y_{t}\left(t_{0}, \phi_{0}\right)=y_{t t}\left(t_{0}, \phi_{0}\right)=0<y_{t t t}\left(t_{0}, \phi_{0}\right)$ and $y_{t \phi}\left(t_{0}, \phi_{0}\right)>0$. Consequently, locally, in a neighborhood of the point $\left(1,0, t_{0}\right)$ of the space of variables $x, y, t$, the surface swept up by the time optimal extremals is defined by the equation $y=Y(t, x)$, where $Y$ is a smooth function, $Y\left(t_{0}, 1\right)=Y_{t}\left(t_{0}, 1\right)=$ $Y_{t t}\left(t_{0}, 1\right)=0 \neq Y_{t t t}\left(t_{0}, 1\right) Y_{t x}\left(t_{0}, 1\right)$. According to Mather's division theorem [9] this equation is locally, in a neighborhood of this point, equivalent to the equation

$$
\left(t-t_{0}\right)^{3}+b(x, y)\left(t-t_{0}\right)^{2}+c(x, y)\left(t-t_{0}\right)+d(x, y)=0,
$$


where $b, c, d$ are smooth functions, $b(1,0)=c(1,0)=d(1,0)=0 \neq c_{x}(1,0)$ $d_{y}(1,0)$. The change of variables (which is an $R^{+}$-equivalence) $\tilde{t}=t-t_{0}+b(x, y) / 3$, $\tilde{x}=c(x, y)-b^{2}(x, y) / 3, \tilde{y}=d(x, y)-b^{3}(x, y) / 27-b(x, y) \tilde{x} / 3$ reduces this equation to the form (we omit the tilde in the notations of new variables) $t^{3}+x t+y=0$. Consequently, the time optimal function has singularity (10) from the list of Theorem 3 at the point $(1,0)$ up to $R^{+}$-equivalence. It is clear that this singularity is stable under small perturbations of the system.

3. Maximum function over a preimage. In this section we first prove Theorems 1-3 using Theorem 4 and then prove the last theorem.

3.1. Proof of Theorems 1-3. It is sufficient to show that the set of pairs for which the assertions of Theorems 1-3 hold true are dense. Indeed, it follows from this and from the definition of the $I \Gamma$-stability that these statements are valid for a generic pair.

Consider a pair $\left(g_{0}, \tau_{0}\right)$. For $m$ equal to 1 or 2 and $n \geq m$ the $L R$-stable mappings $\tau$ form an open dense set in $C_{p}^{\infty}\left(N, R^{m}\right)[9]$. Consequently, the pair $\left(g_{0}, \tau_{0}\right)$ can be approximated by a pair $\left(g_{0}, \tau_{1}\right)$ where $\tau_{1}$ is an $L R$-stable mapping. By virtue of Theorem 4 (for a fixed $L R$-stable mapping $\tau=\tau_{1}$ ) the pair $\left(g_{0}, \tau_{1}\right)$ can be approximated by an $I \Gamma$-stable pair $\left(g_{1}, \tau_{1}\right)$ for which either the statement of Theorem 2 (for $m=1$ ) or that of Theorem 3 (for $m=2$ ) is true. We have thus proved Theorems 1-3 modulo Theorem 4.

3.2. Proof of Theorem 4. The upper boundary of the image of the mapping of the pair is the union of all points $(t, q)$ of this image such that for any $t_{1}>t$ the point $\left(t_{1}, q\right)$ does not belong to the image. The following lemma and its corollary are obvious.

LEMMA 1. The graph of the maximum function over the preimage coincides with the upper boundary of the image of the mapping of the pair.

COROLlary 1. If either the statement of Theorem 2 (for $m=1$ ) or that of Theorem $3($ for $m=2)$ is valid for an I $\Gamma$-stable pair then this statement is also true for any pair sufficiently close to it.

By virtue of this corollary, in order to prove Theorem 4 (both for fixed $L R$ stable mapping $\tau$ and for fixed function $g$ with Morse critical points) it is sufficient to show that in the corresponding set of pairs the $I \Gamma$-stable pairs for each of which either the statement of Theorem 2 (for $m=1$ ) or that of Theorem 3 (for $m=2$ ) holds true, are dense.

The pair $\left(g_{0}, \tau_{0}\right)$ with $\tau_{0} L R$-stable can be approximated by a pair $\left(g, \tau_{0}\right)$ such that the function $g$ has only Morse critical points since such functions form an open dense set in $C^{\infty}(N, R)$. By virtue of Theorem 4 , for fixed $g$ with Morse critical points the pair $\left(g, \tau_{0}\right)$ can be approximated by an $I \Gamma$-stable pair $(g, \tau)$ for which either the statement of Theorem 2 (for $m=1$ ) or that of Theorem 3 (for $m=2)$ is valid. It follows from this and from the $L R$-stability of $\tau_{0}$ that $\left(g_{0}, \tau_{0}\right)$ 
can be approximated by an $I \Gamma$-stable pair $\left(\tilde{g}, \tau_{0}\right)$ for which either the statement of Theorem 2 (for $m=1$ ) or that of Theorem 3 (for $m=2$ ) is true. Thus the statement of Theorem 4 for fixed $L R$-stable mapping $\tau$ follows from the statement of this theorem for fixed $g$ with Morse critical points. Let us prove the latter fact.

Throughout this section the function $g$ with Morse critical points is fixed. We must show the density $C_{p}^{\infty}\left(N, R^{m}\right)$ of the set of mappings $\tau$ for which the pair $(g, \tau)$ is $I \Gamma$-stable and either the statement of Theorem 2 (for $m=1$ ) or that of Theorem 3 (for $m=2$ ) is valid. We shall only investigate the case $n>m=2$. In the other two cases, i.e. $n \geq m=1$ and $n=m=2$, the arguments are similar. We shall first formulate a number of auxiliary statements.

In the jet space $J_{p}^{4}\left(N, R^{2}\right)$ (of mappings $\tau$ ) we denote by $Q_{1}$ the set of jets associated with a singular point of $\tau$ which is not (stably $L R$-) equivalent to a singular point of Whitney fold or gather type (cusp). By $Q_{2}$ we denote the set of jets associated with a singular point of the mapping $(g, \tau)$ either of corank larger than 1 , or of corank equal to 1 at which the corank of the quadratic differential exceeds 1 . Denote by $\Sigma^{I}(\phi)$ Bordmann's manifold with index $I$ in the jet space of mappings $\phi$, by $j^{k} \phi$ the $k$-jet extension of the mapping $\phi$, and by $\alpha$ the standard projection of the jet space onto the preimage. Set

$$
\begin{gathered}
Q=Q_{1} \cup Q_{2} ; \quad \Sigma=\left\{\xi \in J_{p}^{4}\left(N, R^{2}\right) \backslash Q \mid j^{4}(g, \tau)(\alpha(\xi)) \in \Sigma^{1}(g, \tau)\right\} ; \\
\Sigma_{\tau}^{1}=\left\{\xi \in \Sigma \mid j^{4} \tau(\alpha(\xi)) \in \Sigma^{1}(\tau)\right\} ; \\
\Sigma_{g}^{1}=\left\{\xi \in \Sigma \mid j^{4}(g, \tau)(\alpha(\xi)) \in \Sigma^{1,1}(g, \tau)\right\} ; \\
\Sigma^{1}=\Sigma_{\tau}^{1} \cup \Sigma_{g}^{1} ; \quad \Sigma_{\tau}^{2}=\left\{\xi \in \Sigma_{\tau}^{1} \mid j^{4} \tau(\alpha(\xi)) \in \Sigma^{1,1}(\tau)\right\} ; \quad \Sigma^{3}=\Sigma_{\tau}^{2} \cap \Sigma_{g}^{1} ; \\
\Sigma_{g}^{2}=\left\{\xi \in \Sigma_{g}^{1} \mid j^{4}(g, \tau)(\alpha(\xi)) \in \Sigma^{1,1,1}(g, \tau)\right\} ; \quad \Sigma^{2}=\Sigma_{\tau}^{2} \cup \Sigma_{g}^{2} \cup\left(\Sigma_{\tau}^{1} \cap \Sigma_{g}^{1}\right) ;
\end{gathered}
$$

Here $j^{4} \tau(\alpha(\xi))=\xi$ everywhere. The following lemmas and corollaries are useful.

LEMMA 2. For a mapping $\tau$ in general position,

$$
j^{4} \tau(N) \cap Q=\emptyset .
$$

Lemma 3. $\Sigma$ is a smooth closed submanifold in $j_{p}^{4}\left(N, R^{2}\right) \backslash Q$ of codimension $n-2$.

LEMMA 4. $\Sigma_{\tau}^{1}$ and $\Sigma_{g}^{1}$ are smooth closed submanifolds of the manifold $\Sigma$ of codimension $1, \Sigma_{\tau}^{2}$ (respectively $\Sigma_{g}^{2}$ ) is a smooth closed submanifold of the manifold $\Sigma_{\tau}^{1}$ (respectively $\Sigma_{g}^{1}$ ) of codimension 1 .

LEMMA 5. $\Sigma_{\tau}^{i}$ and $\Sigma_{g}^{1}$ have a transversal intersection in $\Sigma$, and so the intersection is a smooth closed submanifold of $\Sigma_{g}^{1}$ of codimension $i, i=1,2$.

Lemmas $2-5$ will be proved in Subsection 3.3. These lemmas and Thom's transversality theorem [9] imply the following statement.

COROLlary 2. The 4-jet extension of a generic mapping $\tau$ is transversal to each of the manifolds $\Sigma, \Sigma_{\tau}^{1}, \Sigma_{g}^{1}, \Sigma_{\tau}^{2}, \Sigma_{g}^{2}, \Sigma^{3}$, and, in particular, the intersection $j^{4} \tau(N) \cap \Sigma^{3}$ is empty. 
Denote the preimages under $j^{4} \tau$ of the manifolds $\Sigma, \Sigma_{\tau}^{1}, \Sigma_{g}^{1}, \Sigma_{\tau}^{2}, \Sigma_{g}^{2}, \Sigma_{\tau}^{1} \cap$ $\Sigma_{g}^{1}$ and of the sets $\Sigma \backslash \Sigma^{1}, \Sigma^{1} \backslash \Sigma^{2}, \Sigma^{2}$ by $S, S_{\tau}^{1}, S_{g}^{1}, S_{\tau}^{2}, S_{g}^{2}, S_{g, \tau}^{2}, S_{0}, S_{1}, S_{2}$ respectively. The next assertion follows from Lemmas $2-5$ and Corollary 2.

COROllary 3. For a generic mapping $\tau$ the following three statements are valid:

(1) $S$ is a smooth closed submanifold in $N$ of codimension $n-2$;

(2) $S_{g}^{2}$ (respectively $S_{\tau}^{2}$ ) is a smooth closed submanifold of codimension 1 in $S_{g}^{1}$ (respectively $S_{\tau}^{1}$ ) which is itself a smooth closed submanifold in $S$ of codimension 1 ;

(3) $S_{g}^{1}$ and $S_{\tau}^{1}$ have a tranversal intersection in $S$ and $S_{g, \tau}^{2} \cap\left(S_{g}^{2} \cup S_{\tau}^{2}\right)=\emptyset$.

Fix a stratification on $S$ with strata $S_{0}, S_{1}$, and $S_{2}$.

Definition ([9]). Suppose that $H_{1}, H_{2}, \ldots, H_{k}$ are the strata of the stratified manifold $H$, and $\phi: H \rightarrow R^{l}$ is a differentiable mapping. The mapping $\phi$ satisfies the condition of normal intersection on this manifold if for any pairwise distinct points $z^{i} \in H_{j(i)}$ whose images under the mapping $\phi$ are the same, the subspaces $(d \phi)\left(z^{i}\right) T_{z^{i}} H_{j(i)}, j(i) \in\{1, \ldots, k\}, 1 \leq i \leq r$, are in general position in the tangent space to $R^{l}$ at the point $\phi\left(z^{1}\right)$.

LEMMA 6. For a generic mapping $\tau$ the mapping $(g, \tau)$ satisfies the condition of normal intersection on the stratified manifold $S$.

Lemma 6 will be proved in Subsection 3.4. By the lemma, for a generic mapping $\tau$ there can only occur double or triple self-intersections of the image of the stratum $S_{0}$ in the image of the manifold $S$ under the mapping $(g, \tau)$, or intersections of the images of the strata $S_{0}$ and $S_{1}$. All these intersections are transversal. Let $S_{00}, S_{000}$ and $S_{01}$ be the sets defined by these intersections, respectively. Denote the remaining parts of the images of the strata $S_{0}, S_{1}$ and the image of the stratum $S_{2}$ by the same symbols $\left(S_{0}, S_{1}\right.$ and $S_{2}$ respectively; the meaning will be clear from the context). Fix a stratification with strata $S_{0}, S_{1}, S_{2}, S_{00}, S_{000}, S_{01}$ on the image $(g, \tau)(S)$.

LEMMA 7. For a generic mapping $\tau$ the restriction of the projection $(t, q) \mapsto q$ to the stratified manifold $(g, \tau)(S)$ satisfies the condition of normal intersection on this manifold.

This lemma will be proved in Subsection 3.4.

Take a mapping $\tau$ for which the conclusions of Lemmas 2, 6, 7 and Corollaries 2,3 are true. In order to complete the proof of Theorem $4(n>m=2$ in the case being considered), it is sufficient to show that the pair $(g, \tau)$ is $I \Gamma$-stable and the assertion of Theorem 3 is true for it. We shall carry out the proof in three stages. At the first stage we shall make sure that the mapping $(g, \tau)$ is $L R$-stable, and at the second and third stages we shall verify, respectively, whether the statement of Theorem 3 is valid for it and whether it is $I \Gamma$-stable.

St ag e 1 . The mapping $(g, \tau)$ is proper and the dimension of the image-space is three. Consequently, to prove that this mapping is $L R$-stable it is sufficient to 
make sure that

(1) it has Whitney fold, Whitney gather or swallowtail type singularities, and

(2) it satisfies the condition of normal intersection on the stratification of the set of its singular points with the strata being unions of its singular points of the same type.

Due to Lemma 2 we have $j^{4} \tau(N) \cap Q_{2}=\emptyset$. Hence at every singular point of the pair $(g, \tau)$ its rank is two and the codimension of the quadratic differential of this singular point is smaller than two. Consequently, near every singular point $p$ this pair can be written in one of the following two forms (recall that $g$ is a function with Morse critical points):

$$
\begin{gathered}
g(z)=g(p)+\phi\left(z_{1}, z_{2}\right) \pm z_{3}^{2} \pm \ldots \pm z_{n}^{2}, \quad \tau(z)=\tau(p)+\left(z_{1}, z_{2}\right), \\
g(z)=g(p)+z_{1}, \quad \tau(z)=\tau(p)+\left(z_{2}, \phi\left(z_{1}, z_{2}, z_{3}\right) \pm z_{4}^{2} \pm \ldots \pm z_{n}^{2}\right),
\end{gathered}
$$

where $\phi$ is a smooth function, $\phi(p)=0$, in suitable local coordinates near $p$ with origin at $p$, and, in addition, in the second case, under suitable numbering of the coordinates in the $q$-plane. For the function $g$ the point $p$ is critical in the first case and noncritical in the second one. (It is easy to use these two forms in order to obtain stabilization, after $n=3$, of the list of generic singularities of the maximum function over the preimage for $m=2$. For this purpose, it is sufficient to consider the elementary smooth problem $g(z) \rightarrow$ extr under the constraint $\tau(z)=(x, y)$ and to apply the method of Lagrange multipliers. The extremum can be only attained for $z_{4}=\ldots=z_{n}=0$. Below the statement about the stabilization will follow automatically from the theory of singularities.)

It is easy to see that in the first case $p$ is a Whitney fold singular point of $\tau$.

In the second case $p$ is a nonsingular point of $(g, \tau)$ if $\phi_{z_{3}}(p) \neq 0$, and a singular point of Whitney fold type if $\phi_{z_{3}}(p)=0 \neq \phi_{z_{3} z_{3}}(p)$. If $\phi_{z_{3}}(p)=0=\phi_{z_{3} z_{3}}(p) \neq$ $\phi_{z_{3} z_{3} z_{3}}(p)$, then $j^{4}(g, \tau)(p) \in \Sigma^{1,1,0}(g, \tau)$. Due to Corollary $2, j^{4} \tau \pitchfork \Sigma_{g}^{1}$ and, hence, $j^{4}(g, \tau) \pitchfork_{p} \Sigma^{1,1,0}(g, \tau)\left(\pitchfork_{B}\right.$ means transversality on the set $\left.B\right)$ and $p$ is a singular point of Whitney gather type of $(g, \tau)$. Finally, if $\phi_{z_{3}}(p)=0=\phi_{z_{3} z_{3}}(p)=$ $\phi_{z_{3} z_{3} z_{3}}(p)$ then $p \in S_{2}$. Again due to Corollary $2, j^{4} \tau \pitchfork \Sigma^{2}$, and consequently,

$$
\operatorname{rank}\left|\begin{array}{lll}
\phi_{z_{3} z_{1}} & \phi_{z_{3} z_{2}} & 0 \\
\phi_{z_{3} z_{3} z_{1}} & \phi_{z_{3} z_{3} z_{2}} & 0 \\
\phi_{z_{3} z_{3} z_{3} z_{1}} & \phi_{z_{3} z_{3} z_{3} z_{2}} & \phi_{z_{3} z_{3} z_{3} z_{3}}
\end{array}\right|(p)=3 \text {. }
$$

Thus, $\phi_{z_{3} z_{3} z_{3} z_{3}}(p) \neq 0, j^{4}(g, \tau) \in \Sigma^{1,1,1,0}(g, \tau), j^{4}(g, \tau) \pitchfork \Sigma^{1,1,1,0}(g, \tau)$ and the mapping $(g, \tau)$ has a swallowtail singularity at the point $p$. So the mapping $(g, \tau)$ has only critical points of Whitney fold, Whitney gather or swallowtail type. Singular points of these types divide $S$ into strata $S \backslash S_{g}^{1}, S_{g}^{1} \backslash S_{g}^{2}$ and $S_{g}^{2}$ respectively. It follows from Lemma 6 that the pair $(g, \tau)$ satisfies the condition of normal intersection on this stratification of the manifold $S$.

Thus the pair $(g, \tau)$ is $L R$-stable.

We have also proved the following statement. 
LEMMA 8. Each of the critical points of the mapping $(g, \tau)$ is a critical point of one of the following three types: Whitney's fold, Whitney's gather or a swallowtail, provided the assertions of Lemma 2 and Corollary 2 are true for the mapping $\tau$.

$\mathrm{St}$ ag e 2. We have to show that the statement of Theorem 3 is valid for the pair $(g, \tau)$. Let $A=\left(t_{0}, q_{0}\right)$ be a point on the graph of the corresponding maximum function over the preimage. The preimage $P=(g, \tau)^{-1}(A)$ does not contain a singular point of $\tau$ of Whitney gather type since the image of such a point belongs to the interior of the whole image $(g, \tau)(N)$. Consequently, $P \cap\left(S_{g}^{1} \backslash S_{g}^{2}\right)=\emptyset$. Two cases are possible here: the intersection $P \cap S_{\tau}^{1}$ is either (1) empty or (2) not empty.

In the first case the point $A$ belongs to one of four subsets of the stratified manifold $(g, \tau)(S)$, namely, (a) $S_{0}$, (b) $S_{00}$, (c) $S_{000}$, (d) $S_{g}^{2}$. The germ at the point $A$ of this manifold is the germ at this point, respectively, (a) of a smoothly embedded surface passing through it; (b) of two or (c) three smoothly embedded surfaces transversally intersecting at this point, (d) of a swallowtail surface with vertex at this point. The tangent set to this manifold does not have a vertical direction (i.e. the direction of the $t$-axis) since $P \cap S_{\tau}^{1}=\emptyset$. Consequently, the germ $\left(G, q_{0}\right)$ is $\Gamma$-equivalent to the germ at zero of functions (1), (2), (5), (11), respectively, of the list in Theorem 3. These singularities are precisely those of the maximum function of a generic two-parameter family of functions. The $R^{+}-$ equivalence is sufficient to reduce them to the respective normal forms [6].

In the second case $P \cap S_{\tau}^{1} \neq \emptyset$. The point $A$ belongs to one of three strata of the image $(g, \tau)(S)$, namely, (a) $S_{2}$, (b) $S_{1}$, (c) $S_{01}$. In subcases (a) and (b) the set $P$ consists of one point $p$ which belongs to $S_{\tau}^{2} \backslash S_{g}^{1}$ (since $P \cap\left(S_{g}^{1} \backslash S_{g}^{2}\right)=\emptyset$ ) and $S_{\tau}^{1} \backslash S_{\tau}^{2}$ respectively. In particular, $p$ is a noncritical point of the function $g$ and a singular point of Whitney fold type of the mapping $(g, \tau)$. Consequently, in both subcases, near $p$ this mapping can be written as

$$
t=z_{1}, \quad x=z_{2}, \quad y=\phi\left(z_{1}, z_{2}\right)+\varepsilon_{3} z_{3}^{2}+\ldots+\varepsilon_{n} z_{n}^{2},
$$

where $\varepsilon_{i}= \pm 1, \phi$ is a smooth function, $\phi(p)=0=\phi_{z_{1}}(p)$, in suitable local coordinates $z_{1}, z_{2}, \ldots, z_{n}$ in the preimage-space near the point $p$ with origin at it and in suitable coordinates $t, x=x(q), y=y(q)$ in the image-space near the point $A$ with origin at $A$. (Note that all $\varepsilon_{i}$ are identical and their sign is the same as the sign of the first nonzero $z_{1}$-derivative of $\phi$ at $p$, otherwise we would have $G\left(q_{0}\right)>t_{0}$.) On the manifold $S$ we have $\operatorname{rank}(g, \tau)^{\prime} \equiv 2$ and, consequently, $z_{3}=z_{4}=\ldots=z_{n}=0$. Thus, near the point $A$, the graph of the function $G$ lies on the surface $y=\phi(t, x)$. Due to the choice of $\tau$ we have $j^{4} \tau(N) \cap Q_{1}=\emptyset$. This implies that every critical point of the mapping $\tau$ is either a Whitney fold or a Whitney gather. From this and from the definition of the manifolds $S_{\tau}^{2}$ and $S_{\tau}^{1}$ we find that in subcases (a) and (b) the point $p$ is a Whitney gather or Whitney fold point of $\tau$ respectively. Thus we have $\phi_{z_{1} z_{1}}(p)=0 \neq \phi_{z_{1} z_{1} z_{1}}(p) \phi_{z_{1} z_{2}}(p)$ and $\phi_{z_{1} z_{1}}(p) \neq 0$ respectively. Applying Mather's division theorem [9] we find 
that the equation $y=\phi(t, x)$ is locally near $A$ equivalent to the equation $t^{3}+$ $a(x, y) t^{2}+b(x, y) t+c(x, y)=0$ in subcase (a) and to the equation $t^{2}+d(x, y) t+$ $e(x, y)=0$ in subcase (b), where $a, b, c, d, e$ are smooth functions vanishing at zero, $b_{x}(0,0) c_{y}(0,0) \neq 0 \neq e_{y}(0,0)$. Setting $t=t+a(x, y) / 3, \tilde{x}=b(x, y)-a^{2}(x, y) / 3$, $\tilde{y}=c(x, y)-a^{3}(x, y) / 27-\tilde{x} a(x, y) / 3$ in subcase $(\mathrm{a})$ and $\tilde{t}=t+d(x, y) / 2, \tilde{x}=$ $d^{2}(x, y) / 4-e(x, y), \tilde{y}=x$ in subcase $(\mathrm{b})$, we reduce these equations to the forms (we omit the tildes) $t^{3}+x t+y=0$ and $t^{2}-x=0$ respectively. Consequently, in subcase (a) the germ $\left(G, q_{0}\right)$ is $\Gamma$-equivalent (and $R^{+}$-equivalent) to the germ at zero of function (10) in the list of Theorem 3.

In subcase (b) the singularity of the function $G$ at the point $q_{0}$ depends only on whether there are points of the set $(g, \tau)(S)$ different from $A$ on the straight line $q=q_{0}$. If there are no such points then evidently the germ $\left(G, q_{0}\right)$ is $\Gamma$-equivalent to the germ at zero of function (3) in the list of Theorem 3. Now if there are points of this kind we take one, say $A_{1}$, that is closest to $A$. In the stratified manifold $(g, \tau)(S)$ the sum of the codimensions of the strata containing these two points is less than three since by Lemma 7 the restriction of the projection $(t, q) \mapsto q$ onto this manifold satisfies the condition of normal intersection on it. Consequently, $A_{1}$ belongs to $S_{0}, S_{00}$ or $S_{1}$. When $A_{1} \in S_{0}$ the germ $\left(G, q_{0}\right)$ is evidently $\Gamma$-equivalent to the germ at zero of function (4) in the list of Theorem 3.

When $A_{1} \in S_{00}$ the germ $\left((g, \tau)(S), A_{1}\right)$ is the germ at $A_{1}$ of two smoothly embedded surfaces transversally intersecting at $A_{1}$, and at that point the vertical direction has no contact with any one of these surfaces. Furthermore by Lemma 7 the images of the strata $S_{00}$ and $S_{1}$ under the projection $(t, q) \mapsto q$ are transversal at $q_{0}$. Hence we find that when $A_{1} \in S_{00}$ the germ $\left(G, q_{0}\right)$ is $\Gamma$-equivalent to the germ at zero of function (6) in the list of Theorem 3.

If $A_{1} \in S_{1}$, then, by Lemma 7 we have: (1) the image of the stratum $S_{1}$ under the projection $(t, q) \mapsto q$ has a transversal self-intersection at $q_{0}$ and (2) any point of $\left\{q=q_{0}\right\} \cap(g, \tau)(S)$ different from $A$ and $A_{1}$ lies in $S_{0}$. It is easy to see that depending on whether the last intersection is empty or not, the germ $\left(G, q_{0}\right)$ is $\Gamma$-equivalent to the germ at zero of functions (7) and (8) respectively in the list of Theorem 3.

In subcase (c) (when $A \in S_{01}$ ) the set $P$ consists of two points $p_{1} \in S_{\tau}^{1} \backslash S_{\tau}^{2}$ and $p_{2} \in S_{0}$. As in subcase (b), we reduce the image of the germ $\left(S, p_{1}\right)$ under the mapping $(g, \tau)$ to the germ at zero of the surface $t^{2}=x$ by means of a suitable choice of coordinates $t, x=x(q), y=y(q)$ in the image-space with origin at $A$. Then the image of the germ $\left(S, p_{2}\right)$ under this mapping will be reduced to the germ at zero of the surface $t=a(x, y)$, where $a$ is a smooth function, $a(0,0)=0$ since $p_{2} \in S_{0}$ and $(g, \tau)\left(p_{2}\right)=A$. We have $a_{y}(0,0) \neq 0$ since by Lemma 6 the images of the strata $S_{0}$ and $S_{1}$ under the mapping $(g, \tau)$ intersect transversally. Setting $\tilde{y}=a(x, y)$ and omitting the tilde, we find that the germ $\left(G, q_{0}\right)$ is $\Gamma$-equivalent ( $R^{+}$-equivalent) to the germ at zero of function $(9)$ in the list of Theorem 3.

Thus the statement of Theorem 3 is true for the pair $(g, \tau)$. 
St a g e 3. To prove the $I \Gamma$-stability of the pair $(g, \tau)$ it is sufficient to prove the $I \Gamma$-stability of the boundary of its image since this pair is $L R$-stable. For $q_{0} \in \tau(N)$ the intersection of the straight line $q=q_{0}$ with this boundary (and with the set $(g, \tau)(S))$ consists of a finite number of points since the mapping $\tau$ is proper and every critical point of its restriction to the manifold $S$ is either a Whitney fold or a Whitney gather. By Lemma 7, the sum over these points of codimensions (in the stratified manifold $(g, \tau)(S)$ ) of the strata containing them is less than three. Then, besides the finite number of points of the stratum $S_{0}$, this intersection may contain either (1) exactly one point of one of the strata $S_{1}, S_{00}, S_{01}, S_{000}, S_{2}$, or (2) two distinct points from the union of the strata $S_{1}$ and $S_{00}$. It follows that in order to prove the $I \Gamma$-stability it is sufficient to prove the $I \Gamma$-stability, in the first case, of singularities $(3),(2),(9),(5),(10),(11)$ of the list in Theorem 3 respectively, and, in the second case, either singularities (6) and (7) of this list or the tranversal overlapping of two singularities of type (2) of this list (transversal overlapping means that (a) it takes place on different levels with respect to $t$ and (b) the images under the projection $(t, q) \mapsto q$ of the curves of singular points of these two singularities have transversal intersection at the point $\left.q_{0}\right)$. It is easy to see that when the pair $(g, \tau)$ is $L R$-stable, the indicated singularities are also $I \Gamma$-stable.

This completes the proof of Theorem 4.

3.3. Proofs of Lemmas 2-5. Let us prove Lemma 2. A generic mapping $\tau$ is $L R$-stable and each of its critical points is either a Whitney fold or a Whitney gather. Then, for such a mapping $j^{4} \tau(N) \cap Q_{1}=\emptyset$. The set $Q_{2}$ is a closed Whitney stratified submanifold in $j_{p}^{4}\left(N, R^{2}\right)$ of codimension larger than $n$. Consequently, by virtue of Thom's transversality theorem, $j^{4} \tau(N) \cap Q_{2}=\emptyset$ for a generic mapping $\tau$. Thus we have $j^{4}(N) \cap\left(Q_{1} \cup Q_{2}\right)=\emptyset$ for a generic mapping $\tau$. We have proved Lemma 2 since $Q=Q_{1} \cup Q_{2}$.

It is sufficient to prove the statements of Lemmas $4-5$ in the jet space over some chart on $N$. Without loss of generality, we may assume $N=R_{z}^{n}, z=$ $\left(z_{1}, z_{2}, \ldots, z_{n}\right), \xi \in j^{4}\left(R_{z}^{n}, R^{2}\right), j^{4} \tau(\alpha(\xi))=\xi$.

The set $\Sigma$ is closed in $j^{4}\left(R_{z}^{n}, R^{2}\right) \backslash Q$ and is defined by the system of equations

$$
\operatorname{det}\left|\begin{array}{lll}
g_{z_{i}} & g_{z_{j}} & g_{z_{k}} \\
\tau_{1, z_{i}} & \tau_{1, z_{j}} & \tau_{1, z_{k}} \\
\tau_{2, z_{i}} & \tau_{2, z_{j}} & \tau_{2, z_{k}}
\end{array}\right|=0, \quad 1 \leq i<j<k \leq n .
$$

Near every point $\xi \in \Sigma$ there are exactly $n-2$ independent equations among them since $\operatorname{rank}(g, \tau)^{\prime}(\alpha(\xi))=2$. Consequently, $S$ is a smooth closed submanifold in $j^{4}\left(R_{z}^{n}, R^{2}\right)$ of codimension $n-2$. We have proved Lemma 3 .

For $\xi \in \Sigma^{1}$ the point $\alpha(\xi)$ is not a critical point of the function $g$. Indeed, if $g^{\prime}(\alpha(\xi))=0$ then, first, we have $\operatorname{rank} \tau^{\prime}(\alpha(\xi))=2 \operatorname{since} \operatorname{rank}(g, \tau)^{\prime}(\alpha(\xi))=2$, and, second, $\alpha(\xi)$ is a critical point of Whitney fold type of the mapping $(g, \tau)$ since the function $g$ has only Morse critical points. Thus $\xi \notin \Sigma_{\tau}^{1}$ and $\xi \notin \Sigma_{g}^{1}$. 
Consequently, $\xi \notin \Sigma^{1}$, and we have a contradiction.

For the jet $\xi \in \Sigma^{1}$ we choose coordinates $z_{1}, z_{2}, \ldots, z_{n}$ in $R_{z}^{n}$ such that $\tau_{z_{2}}(\alpha(\xi)) \neq 0$ and $g(z) \equiv z_{1}$ near the point $\alpha(\xi)$. For definiteness we assume that $\tau_{1, z_{2}}(\alpha(\xi)) \neq 0$. In these coordinates the manifold $\Sigma$ is defined near the point $\xi$ by a system of $n-2$ independent equations $\psi_{i}=0,2<i \leq n$, where $\psi_{i}=\tau_{2, z_{i}}-\tau_{1, z_{i}} \tau_{2, z_{2}} / \tau_{1, z_{2}}$.

If the jet $\xi$ belongs to the set $\Sigma_{\tau}^{1}$ which is "cut out" of $\Sigma$ by the equation $\psi_{1}=0$ or is defined by a system of $n-1$ independent equations $\psi=0$, where $\psi=\left(\psi_{1}, \psi_{3}, \ldots, \psi_{n}\right)$. Consequently, $\Sigma_{\tau}^{1}$ is a smooth closed submanifold in $\Sigma$ of codimension 1. The set $\Sigma_{\tau}^{2}$ is defined on the manifold $\Sigma_{\tau}^{1}$ by the equation $\operatorname{det}\left(\partial \psi / \partial z^{\prime}\right)=0$, where $z^{\prime}=\left(z_{1}, z_{3}, \ldots, z_{n}\right)$. This equation is nondegenerate since for the jet $\xi \in \Sigma_{\tau}^{1}$ we have $\operatorname{rank}\left(\partial \psi / \partial z^{\prime}\right)>n-2$. Evidently it does not depend on the equations $\psi=0$. Consequently, $\Sigma_{\tau}^{2}$ is a smooth closed submanifold in $\Sigma_{\tau}^{1}$ of codimension 1 .

If the jet $\xi$ belongs to the set $\Sigma_{g}^{1}$ then near $\xi$ this set is "cut out" of $\Sigma$ by the equation $\Delta=0$, where $\Delta=\operatorname{det} \partial \tilde{\Psi} / \partial \tilde{z}, \tilde{\Psi}=\left(\Psi_{3}, \Psi_{4}, \ldots, \Psi_{n}\right), \tilde{z}=\left(z_{3}, z_{4}, \ldots, z_{n}\right)$. This equation can be solved for some derivative $\tau_{2, z_{i}, z_{j}}$ since for the jet $\xi \in \Sigma$ we have $\operatorname{rank}(\partial \tilde{\Psi} / \partial \tilde{z})>n-4$. It does not depend on the equations $\tilde{\psi}=0$ since the latter do not include the second derivatives of $\tau$. Consequently, $\Sigma_{g}^{1}$ is a smooth closed submanifold in $\Sigma$ of codimension 1 .

The submanifold $\Sigma_{\tau}^{i}$ intersects the submanifold $\Sigma_{g}^{1}$ transversally in $\Sigma$ since on $\Sigma$ the equations defining them are independent. Consequently, the intersection $\Sigma_{\tau}^{i} \cap \Sigma_{g}^{1}$ is a closed smooth submanifold of $\Sigma$ of codimension $i$.

Finally, $\Sigma_{g}^{2}$ is a smooth closed submanifold in $\Sigma_{g}^{1}$ of codimension 1 since for fixed $z_{1}$ these sets are Bordmann's manifolds with indices $(1,1,1)$ and $(1,1)$, respectively, in the jet space of mappings $\left(z_{2}, \ldots, z_{n}\right) \mapsto \tau\left(z_{1}, z_{2}, \ldots, z_{n}\right)$.

We have proved Lemmas 4 and 5 .

3.4. Proofs of Lemmas 6 and 7 . Take multi-indices $I=\left(i_{1}, \ldots, i_{k}\right), J=$ $\left(j_{1}, \ldots, j_{l}\right)$, where $i_{r}, j_{s} \in\{0,1,2,3\}, 1 \leq r \leq k, 1 \leq s \leq l$. Define the sets $\Sigma_{I}=\left\{\left(\xi_{1}, \ldots, \xi_{k}\right) \in j_{k}^{4}\left(N, R^{2}\right) \mid \xi_{r} \in \Sigma^{i_{r}},(g, \tau)\left(\alpha\left(\xi_{r}\right)\right)=(g, \tau)\left(\alpha\left(\xi_{i}\right)\right), 1 \leq r \leq\right.$ $i \leq k\}$, and, for $|I|+k>1$ and $|J|+l>1$, the sets $\Sigma_{I ; J}=\left\{\left(\xi_{1}, \xi_{2}, \ldots, \xi_{k+l}\right) \in\right.$ $\left.J_{k+l}^{4}\left(N, R^{2}\right) \mid\left(\xi_{1}, \ldots, \xi_{k}\right) \in \Sigma_{I},\left(\xi_{k+1}, \ldots, \xi_{k+l}\right) \in \Sigma_{J}, \tau\left(\alpha\left(\xi_{1}\right)\right)=\tau\left(\alpha\left(\xi_{k+1}\right)\right)\right\}$. Each of these sets is a Whitney stratified submanifold in the enveloping space of multijets of codimensions $|I|+k(n+1)-3$ and $|I|+|J|+(k+l)(n+1)-4$, respectively. Here $|I|=i_{1}+\ldots+i_{k},|J|=j_{1}+\ldots+j_{l}$. Due to Lemmas 2-5, the boundary of this submanifold in this space lies in a set which does not meet the image of the multiple 4-jet extension of a generic mapping $\tau$. Now let us first prove Lemma 6 and then Lemma 7.

Due to Lemma 2, Corollary 2 and Lemma 8, a generic pair $(g, \tau)$ has only critical points of Whitney fold, Whitney gather and swallowtail types. It follows that for generic $\tau$ the mapping $j_{k}^{4} \tau$ is transversal to $\Sigma_{I}$ near the diagonal. Consequently, by virtue of the multijet transversality theorem, for generic $\tau$ the mapping $j_{k}^{4} \tau$ is 
transversal to $\Sigma_{I}, k>1$. In particular, $j_{k}^{4} \tau(N) \cap \Sigma_{I}=\emptyset$ if $k n<|I|+k(n+1)-3$ or $|I|+k>3$. Thus for generic $\tau$ the image of its multiple 4 -jet extension can intersect (transversally) only the manifold $\Sigma_{0,0}, \Sigma_{0,0,0}$ and $\Sigma_{0,1}\left(\right.$ or $\Sigma_{1,0}$ ) and cannot intersect other manifold $\Sigma_{I}$. We will show that for the pair $(g, \tau)$ this implies the fulfillment of the condition of normal intersection on the stratified manifold $S$. It is sufficient to verify the transversality of the intersections corresponding to the indicated four manifolds. We shall carry out the computations for the manifolds $\Sigma_{0,0,0}$ and $\Sigma_{0.1}$. In the other two cases $\left(\Sigma_{0,0}\right.$ and $\left.\Sigma_{0,1}\right)$ the arguments are similar. To simplify notation we assume that $n=3$.

Let $j_{3}^{4} \tau\left(p_{1}, p_{2}, p_{3}\right) \in \Sigma_{0,0,0}$. The point $p_{i}$ is a noncritical point of the mapping $\tau$ and a critical point of Whitney fold type of the mapping $(g, \tau)$ since $j_{3}^{4} \tau(N) \cap$ $\Sigma_{I}=\emptyset$ for $|I|>0$. Near the point $p_{i}$ we choose a system of coordinates $u_{i}, v_{i}$, $w_{i}$ with centered origin at this point in which the mapping $(g, \tau)$ has the form $t=\phi_{i}\left(u_{i}, v_{i}\right) \pm w_{i}^{2}, x=u_{i}, y=v_{i}$, where $\phi_{i}$ is a smooth function, $\phi_{i}(0,0)=$ $0, i=1,2,3, \phi_{1} \equiv 0$, in suitable coordinates $t, x(q), y(q)$ in the image-space with origin at the point $(g, \tau)\left(p_{1}\right)$. From the condition $j_{3}^{4} \tau \pitchfork \Sigma_{0,0,0}$ we have $\left(\phi_{2, u} \phi_{3, v}-\phi_{2, v} \phi_{3, u}(0,0) \neq 0\right.$. Consequently, the tangent planes to the graphs $t=\phi_{i}(x, y), i=1,2,3$, at zero are in general position. Hence, at the points $p_{1}$, $p_{2}, p_{3}$ of the stratum $S_{0}$ the condition of normal intersection is fulfiled for the pair $(g, \tau)$.

Let $j_{2}^{4} \tau\left(p_{1}, p_{2}\right) \in \Sigma_{1,0}$. The point $p_{2}$ is a critical point of the mapping $(g, \tau)$ of Whitney fold type and a regular point of the mapping $\tau$ since $j_{2}^{4} \tau(N) \cap \Sigma_{1,1}=\emptyset$. The point $p_{1}$ is either (1) a critical point of Whitney fold type both of $\tau$ and $(g, \tau)$, or (2) a critical point of Whitney gather type of $(g, \tau)$ and a regular point of $\tau$ since $j_{2}^{4} \tau(N) \cap \Sigma_{2,0}=\emptyset$. As in the previous case, in suitable coordinate systems (with origins at the points $\left.p_{1}, p_{2},(g, \tau)\left(p_{1}\right)\right)$ the mapping $(g, \tau)$ can be written as $t= \pm w_{2}^{2}, x=u_{2}, y=v_{2}$ near the point $p_{2}\left(\phi_{2} \equiv 0\right)$ and in one of the following two forms: (1) $t=u_{1}, x=v_{1}, y=\phi_{1}\left(u_{1}, v_{1}, w_{1}\right)$ and (2) $t=\phi_{1}\left(u_{1}, v_{1}, w_{1}\right), X=u_{1}$, $Y=v_{1}$, where $\phi_{1}$ is a smooth function, $\phi_{1}(0,0,0)=\phi_{1, w_{1}}(0,0,0)=0$ and, respectively, (1) $\phi_{1, w_{1} w_{1}}(0,0,0) \neq 0$ and $(2) \phi_{1, w_{1} w_{1}}(0,0,0)=0 \neq \phi_{1, w_{1} w_{1} w_{1}}(0,0,0)$, near the point $p_{1}$. In these two subcases the condition $j_{2}^{4} \tau \pitchfork \Sigma_{0,1}$ implies, respectively, (1) $\left(\phi_{1, w_{1} v_{1}} \phi_{1, u_{1} w_{1}}-\phi_{1, w_{1} w_{1}} \phi_{1, u_{1} v_{1}}\right)(0,0,0) \neq 0$ and $(2)\left(\phi_{1, v_{1}} \phi_{1, u_{1} w_{1}}-\right.$ $\left.\phi_{1, u_{1}} \phi_{1, w_{1} v_{1}}\right)(0,0,0) \neq 0$. Consequently, at zero the subspaces $(d(g, \tau))\left(p_{1}\right) T_{p} S_{1}$ and $(d(g, \tau))\left(p_{2}\right) T_{p} S_{0}$ are in general position, and at the points $p_{1} \in S_{1}$ and $p_{2} \in S_{0}$ the condition of normal intersection is fulfilled for the pair $(g, \tau)$.

We have proved Lemma 6 .

Now we prove Lemma 7 . Every point of the stratum $S_{0}$ of the stratified manifold $(g, \tau)(S)$ is a regular point of the restriction of the projection $(t, q) \mapsto q$ to this manifold. Consequently, in order to prove the lemma, it is sufficient to show that the condition of normal intersection is fulfilled on the stratified manifold $(g, \tau)(S)$ with strata $S_{1}, S_{2}, S_{00}, S_{000}$ and $S_{01}$.

By virtue of Lemma 6 , for generic $\tau$, the pair $(g, \tau)$ satisfies the condition of normal intersection on the stratified manifold $S$. It follows that locally, in 
a sufficiently small neighborhood $U$ of every point of the stratified manifold $(g, \tau)(S) \backslash S_{0}$, the images under the projection $(t, q) \mapsto q$ of any two different points of the set $U \cap\left\{(g, \tau)(S) \backslash S_{0}\right\}$ are distinct. Consequently, for generic $\tau$ the intersection of the set $j_{k+l}^{4} \tau(N) \cap \Sigma_{I, J}$ with a sufficiently small neighborhood of the diagonal is empty. By virtue of Lemmas 2-5, the intersection of this set with a sufficiently small neighborhood of the boundary of $\Sigma_{I, J}$ in $j_{k+l}^{4} \tau\left(N, R^{2}\right)$ is also empty. From this and from the multijet transversality theorem we find that for a generic mapping $\tau$ the mapping $j_{k+l}^{4} \tau$ is transversal to $\Sigma_{I, J}$. In particular, $j_{k+l}^{4} \tau(N) \cap \Sigma_{I, J}=\emptyset$ if $(k+l) n<|I|+|J|+(k+l)(n+1)-4$ or $|I|+|J|+k+l>4$. But $|I|+k>1$ and $|J|+l>1$. Consequently, for generic $\tau$ the image of its multiple 4 -jet extension can intersect (transversally) only the manifolds $\Sigma_{1,1}, \Sigma_{00,1}$ or $\Sigma_{1,00}, \Sigma_{00,00}$ and cannot intersect any other manifold $\Sigma_{I, J}$. We shall show that this implies the fulfillment of the condition of normal intersection for the restriction of the projection $(\tau, q) \mapsto q$ to the stratified manifold $(g, \tau)(S) \backslash S_{0}$. It is sufficient to verify the transversality of the intersections corresponding to the four indicated manifolds. We shall carry out the computations for the manifold $\Sigma_{00,1}$. In the other three cases the computations are similar. To simplify notation, we assume that $n=3$.

Let $j_{3}^{4} \tau\left(p_{1}, p_{2}, p_{3}\right) \in \Sigma_{00,1}$. Each of the points $p_{1}, p_{2}$ is a critical point of Whitney fold type of the mapping $(g, \tau)$ and a regular point of the mapping $\tau$ since $j_{3}^{4} \tau(N) \cap \Sigma_{10,1} \neq \emptyset$. The point $p_{3}$ is either (1) a critical point of Whitney fold type of both $\tau$ and $(g, \tau)$, or (2) a critical point of Whitney gather type of $(g, \tau)$ and a regular point of $\tau$ since $j_{3}^{4} \tau(N) \cap \Sigma_{00,2}=\emptyset$. In appropriate systems of local coordinates $u_{i}, v_{i}, w_{i}$ in the preimage-space with origin at the points $p_{i}$, $i=1,2,3$, and in the local coordinates $t, x(q), y(q)$ and $\tilde{t}, x(q), y(q)$ in the imagespace with origins at the points $(g, \tau)\left(p_{1}\right)$ and $(g, \tau)\left(p_{2}\right)$ respectively, the mapping $(g, \tau)$ can be written as $(t, x, y)=\left(\phi_{i}\left(u_{i}, v_{i}\right) \pm w_{i}^{2}, u_{i}, v_{i}\right)$ near the point $p_{i}, i=$ $1,2,3$, and, correspondingly, $(1)(\tilde{t}, x, y)=\left(u_{3}, v_{3}, \pm u_{3}^{2} \pm w_{3}^{2}\right)$ and $(2)(\tilde{t}, x, y)=$ $\left(w_{3} \phi_{3}\left(u_{3}, v_{3}, w_{3}\right), u_{3}, v_{3}\right)$. Here $\phi_{i}$ are smooth functions, $\phi_{1} \equiv 0=\phi_{2}(0,0,0)=$ $\phi_{3}(0,0,0)=\phi_{3, w_{3}}(0,0,0) \neq \phi_{3, w_{3} w_{3}}(0,0,0)$. The condition $j_{3}^{4} \tau \pitchfork \Sigma_{00,1}$ implies, respectively, (1) $\phi_{2, u_{2}} \neq 0$ and $(2)\left(\phi_{2, u_{2}} \phi_{3, v_{3}}-\phi_{2, v_{2}} \phi_{3, u_{3}}\right)(0,0,0) \neq 0$. In both cases this means that at the points $(g, \tau)\left(p_{1}\right) \in S_{00}$ and $(g, \tau)\left(p_{3}\right) \in S_{1}$ the condition of normal intersection is fulfilled for the restriction of the projection $(t, q) \mapsto q$ to the stratified manifold $(g, \tau)(S) \backslash S_{0}$.

We have proved Lemma 7.

\section{References}

[1] V. I. Arnol'd, On the theory of envelopes, Russian Math. Surveys 31 (3) (1976).

[2] - Singularities in calculus of variations, J. Soviet Math. 27 (1984).

[3] — Catastrophe theory, 2nd ed., Springer, 1988.

[4] V. I. Arnol'd, S. M. Gusein-Zade and A. N. Varchenko, Singularities of Differentiable Maps, 1, Birkhäuser, Basel, 1985. 
[5] V. M. Alekseev, V. M. Tikhomirov and S. V. Fomin, Optimal Control, Nauka, Moscow 1979 (in Russian).

[6] L. N. Bryzgalova, Singularities of the maximum of a parametrically dependent function, Functional Anal. Appl. 12 (1977), 50-51.

[7] A. A. Davydov, Differential inequalities with locally bounded derivatives, Russian Math. Surveys 45 (4) (1990).

[8] J.-P. Dufour, Stabilité simultanée de deux fonctions différentiables, Ann Inst. Fourier (Grenoble), 29 (1) (1979), 263-282.

[9] M. Golubitsky and V. Guillemin, Stable Mappings and Their Singularities, Springer, 1973.

[10] V. I. Matov, Topological classification of the germs of functions of the maximum and minimax of families of functions in general position, Russian Math. Surveys 37 (4) (1982), $127-128$.

[11] B. Morin, Formes canoniques des singularités d'une application différentiable, C.R. Acad. Sci. Paris 260 (1965), 5662-5665, 6503-6506.

[12] L. S. Pontryagin, On the theory of differential games, Uspekhi Mat. Nauk 21 (4) (1961), 219-274 (in Russian).

[13] L. S. Pontryagin, V. G. Boltyanskiǔ, R. V. Gamkrelidze and E. F. Mishchenko, The Mathematical Theory of Optimal Processes, Pergamon Press, New York-Paris 1964

[14] V. M. Zakalyukin, Metamorphoses of fronts, caustics, depending on a parameter; versality of mappings, J. Soviet Math. 27 (1984), 2713-2735. 